\title{
BMJ Open An auditory brainstem implant for treatment of unilateral tinnitus: protocol for an interventional pilot study
}

\author{
Minke J C van den Berge, ${ }^{1}$ Marc J M C van Dijk, ${ }^{\circ}$ Jan D M Metzemaekers, ${ }^{2}$ \\ Bert Maat, ${ }^{\oplus 1}$ Rolien H Free, ${ }^{1}$ Pim van Dijk ${ }^{1}$
}

To cite: van den

Berge MJC, van Dijk MJMC, Metzemaekers JDM, et al. An auditory brainstem implant for treatment of unilateral tinnitus: protocol for an interventional pilot study. BMJ Open 2019;9:e026185. doi:10.1136/ bmjopen-2018-026185

- Prepublication history and additional material for this paper are available online. To view, please visit the journal (http:// dx.doi.org/10.1136/bmjopen2018-026185).

Received 21 August 2018

Revised 4 March 2019

Accepted 11 April 2019
Check for updates

(C) Author(s) (or their employer(s)) 2019. Re-use permitted under CC BY-NC. No commercial re-use. See rights and permissions. Published by BMJ.

\section{${ }^{1}$ Department of}

Otorhinolaryngology/Head \& Neck Surgery, Universitair Medisch Centrum Groningen Groningen, The Netherlands ${ }^{2}$ Department of Neurosurgery, University Medical Center Groningen, Groningen, The Netherlands

Correspondence to

MD Minke J C van den Berge; m.j.c.van.den.berge@umcg.nl

\section{ABSTRACT}

Introduction Tinnitus may have a very severe impact on the quality of life. Unfortunately, for many patients, a satisfactory treatment modality is lacking. The auditory brainstem implant $(\mathrm{ABI})$ was originally indicated for hearing restoration in patients with non-functional cochlear nerves, for example, in neurofibromatosis type II. In analogy to a cochlear implant (Cl), it has been demonstrated that an $\mathrm{ABI}$ may reduce tinnitus as a beneficial side effect. For tinnitus treatment, an $\mathrm{ABI}$ may have an advantage over a $\mathrm{Cl}$, as cochlear implantation can harm inner ear structures due to its invasiveness, while an $A B I$ is presumed to not damage anatomical structures. This is the first study to implant an $A B I$ to investigate its effect on intractable tinnitus.

Methods and analysis In this pilot study, 10 adults having incapacitating unilateral intractable tinnitus and ipsilateral severe hearing loss will have an ABI implanted. The ABI is switched on 6 weeks after implantation, followed by several fitting sessions aimed at finding an optimal stimulation strategy. The primary outcome will be the change in Tinnitus Functioning Index. Secondary outcomes will be tinnitus burden and quality of life (using Tinnitus Handicap Inventory and Hospital Anxiety and Depression Scale questionnaires), tinnitus characteristics (using Visual Analogue Scale, a tinnitus analysis), safety, audiometric and vestibular function. The end point is set at 1 year after implantation. Follow-up will continue until 5 years after implantation.

Ethics and dissemination The protocol was reviewed and approved by the Institutional Review Board of the University Medical Centre Groningen, The Netherlands (METc 2015/479). The trial is registered at www. clinicialtrials.gov and will be updated if amendments are made. Results of this study will be disseminated in peerreviewed journals and at scientific conferences.

Trial registration number NCT02630589.

Trial status Inclusion of first patient in November 2017. Data collection is in progress. Trial is open for further inclusion. The trial ends at 5 years after inclusion of the last patient.

\section{INTRODUCTION}

Tinnitus, which literally means 'ringing in the ears', is defined by the perception of sound or noise in the absence of an external physical sound source. ${ }^{1}$ It is a very common condition (prevalence 5\%-18\% in Western

\section{Strengths and limitations of this study}

- Tinnitus may have a severe impact on the quality of life in the absence of a satisfactory treatment.

- This is the first study to prospectively investigate the effect of the implantation of an auditory brainstem implant for the reduction of incapacitating tinnitus.

- This is a single-centre, non-randomised, interventional pilot study, including 10 patients.

- The treatment effect is measured with validated tinnitus and quality of life questionnaires, as well as safety end points.

- This is a high-risk study because of the invasive surgery; however, it is likely that the potential to ameliorate severely debilitating tinnitus outweighs these risks.

population) and, in a subgroup of patients, it causes extreme distress with far-reaching consequences for daily activities and quality of life. ${ }^{1-3}$ Conventional treatment methods, for example, sound generators and cognitive behavioural therapy, seem not to reduce the loudness of tinnitus, but may improve related depression and quality of life. ${ }^{45}$ However, not all patients benefit from these treatments and there is a remaining group of patients with severe tinnitus for whom there is no conventional treatment modality available. ${ }^{4}$

During the ongoing search for causal treatment methods, it has been demonstrated that a cochlear implant (CI) may be a potential treatment option. In a prospective study, CI implantation in patients with single-sided deafness and tinnitus resulted in significantly reduced tinnitus loudness in the long-term. ${ }^{6}$ However, insertion of an electrode into the cochlea often leads to mechanical damage of intracochlear structures and subsequent, additional hearing loss. Therefore, CI is only indicated in cases where there is severe to profound hearing loss. This means that CI is not an option for the large group of tinnitus patients who still have usable hearing. To fill 
this gap, the auditory brainstem implant (ABI) might be an option.

In 1979, the first ABI was implanted by House and Hitselberger for the purpose of restoring hearing in a patient with neurofibromatosis type II (NF2). ${ }^{78}$ The implant hardware is comparable to that of the CI; however, the ABI was specifically designed to bypass both the cochlea and the auditory nerve to directly stimulate the cochlear nucleus in the brainstem. It is thought that the dorsal cochlear nucleus (DCN) plays an important role in modulation and generation of tinnitus. For example, as a result of increased noise exposure, hyperactivity, expressed as an increased spontaneous activity, can be found in DCN; this in turn reduces residual inhibition and increases excitability. ${ }^{9}$ In an animal model, it was demonstrated that there is behavioural evidence of tinnitus in conditions of increased hyperactivity in the DCN. ${ }^{10}$ Thus, electrical stimulation of the cochlear nucleus in rats led to suppressed behavioural evidence of tinnitus. ${ }^{11}$ This effect might be explained by the possibility that stimulation of DCN compensates the loss of peripheral input caused by noise damage and thereby restores the disturbed balance between excitatory and inhibitory processes. Also, hyperactivity in the DCN might be modulated by direct stimulation of the neuronal circuit and interrupt pathways of hyperactivity to higher regions, such as the inferior colliculus, or it may induce a masking effect. ${ }^{11}$ Several clinical studies have also shown a positive effect of ABI implantation on tinnitus. Soussi and Otto published a study with patients who were implanted with an ABI for the indication of hearing loss. Seven out of 10 patients with tinnitus before the implantation reported a decrease in their tinnitus loudness during stimulation with the ABI. ${ }^{12}$ This finding was confirmed in several other clinical studies, showing a reduction of tinnitus in patients who suffered from tinnitus before ABI implantation after removal of vestibular schwannoma. ${ }^{13-15}$

Together, the preclinical and clinical studies suggest that electrical stimulation of the cochlear nucleus with the ABI may be an effective method to suppress tinnitus. The potential advantage of the ABI over a CI is that it can be implanted without causing hearing damage. Therefore, we designed a pilot study. The objective of this study is to study the effect of the ABI on the suppression of unilateral, incapacitating and intractable tinnitus. We hypothesise that stimulation of the cochlear nucleus by the ABI can reduce tinnitus and, thereby, decrease the tinnitus burden and enhance the quality of life.

\section{METHODS AND ANALYSIS}

\section{Study design}

This is a single-centre, non-randomised, interventional pilot study. The goal is to include 10 patients. There is no control group. The study site is a tertiary academic hospital (University Medical Center Groningen, The Netherlands).

\section{Inclusion criteria}

Adults with unilateral, incapacitating tinnitus that is refractory to conventional treatment methods, are included in this study. Lateralisation (either left or right ear) and the assessment of tinnitus as unilateral was based on patients' perception. The patients must have tinnitus for $>1$ year, with a stable situation over the last year. For the ipsilateral ear, the pure tone audiometry (PTA) thresholds averaged between 1, 2 and $4 \mathrm{kHz}$ must be between 40 and $90 \mathrm{~dB}$. The contralateral ear should have functional hearing ability with PTA thresholds of $<35 \mathrm{~dB}$ (average between 1 , 2 and $4 \mathrm{kHz}$ ), with a minimum of $25 \mathrm{~dB}$ (average between 1,2 and $4 \mathrm{kHz}$ ) difference compared with the tinnitus (ipsilateral) ear.

\section{Exclusion criteria}

Patients with a detectable cause for tinnitus that requires causal therapy, for example, vestibular schwannoma or glomus tumour, are excluded from this study. Also, patients with psychiatric pathology or an unstable psychological situation as declared by a psychiatrist, are excluded. Patients with a life expectancy $<5$ years, a history of blood coagulation pathology, an American Society of Anesthesiologists score $>2$ as well as pregnant women are also excluded from participation. Additionally, anatomic abnormalities that prohibit appropriate placement of the implant, or a history of intolerance to materials used in the implant, are exclusion criteria. An overview of inclusion and exclusion criteria is presented in box 1 .

\section{Study device}

The device used in this study is the Mi1200 SYNCHRONY Auditory Brainstem Implant, manufactured and supplied by MED-EL (Innsbruck, Austria). The ABI is an implantable, electrically active device that consists of a stimulator, a coil with a removable magnet in its centre and an active electrode array that is permanently attached to the stimulator (figure 1). The electrode array stimulates the cochlear nucleus using 12 independent surface electrodes (figure 2). The stimuli are controlled by an external processor that uses stimulation strategies similar to $\mathrm{CI}$.

The intended use of the ABI device is for the electrical stimulation of the cochlear nucleus via an implanted stimulator and a specially designed electrode array to evoke auditory sensations in patients with non-functional cochlear nerves. In this study, the ABI will be primarily investigated for its ability to reduce tinnitus in patients having moderate-to-severe hearing loss despite having a functional cochlear nerve. This is regarded as an off-label use of the ABI, although the surgical method of implantation, the equipment and stimulation strategies are the same as for regular indications.

\section{Recruitment}

Potentially eligible patients are recruited from our outpatient clinic, as well as from our tinnitus database, collected during several years of clinical practice 


\section{Box 1 Inclusion and exclusion criteria.}

\section{Inclusion criteria}

- Unilateral tinnitus.

- Severely incapacitating tinnitus.

- Men or women, age $>18$ years.

- Tinnitus that is present $>1$ year and was stable during the last year.

- Tinnitus that is not responsive to indicated conventional existing treatments (hearing aids and cognitive behavioural therapy). If a psychologist has indicated cognitive behavioural therapy, the patient should have tried this therapy for long enough to reasonably argue that these treatments were not successful. The same applies to the use of hearing aids.

- Ipsilateral ear: pure tone audiometry thresholds $>40 \mathrm{~dB}$ and $<90 \mathrm{~dB}$ (mean between 1, 2 and $4 \mathrm{kHz}$ ).

- Functional hearing in the contralateral ear with pure tone audiometry thresholds $<35 \mathrm{~dB}$ (mean between 1, 2 and $4 \mathrm{kHz}$ ) and with a minimum $\triangle 25 \mathrm{~dB}$ compared with the ipsilateral ear.

- Informed consent after extensive oral and written information about the surgery, complications and uncertain effect of the auditory brainstem implant on tinnitus.

\section{Exclusion criteria}

- Detectable cause for tinnitus that requires causal therapy (eg, vestibular schwannoma, glomus tumour, otosclerosis, arteriovenous malformation) as investigated by radiological and otological examination.

- Psychiatric pathology and/or an unstable psychological situation as declared by a psychiatrist.

- Unrealistic expectations as declared by the investigator and/or psychiatrist.

Life expectancy $<5$ years.

- History of blood coagulation pathology.

- American Society of Anesthesiologists score $>2 .^{25}$

- Pregnancy.

- Anatomic abnormalities that would prevent appropriate placement of the stimulator housing in the bone of the skull.

- Anatomical abnormalities or surgical complications that might prevent placement of the auditory brainstem implant active electrode array.

- Known intolerance to the materials used in the implant (medical grade silicone, platinum, iridium and parylene $\mathrm{C}$ ).

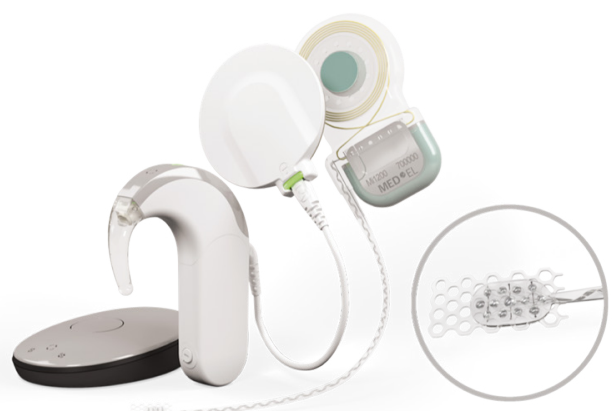

Figure 1 The auditory brainstem implant consists of several components (from left to right): a remote control; the speech processor (consisting of transducer, microphone and connecting cable) which is the external and visible part of the implant; the receiver-stimulator with electrode (implantable component) and close-up of the electrode paddle.

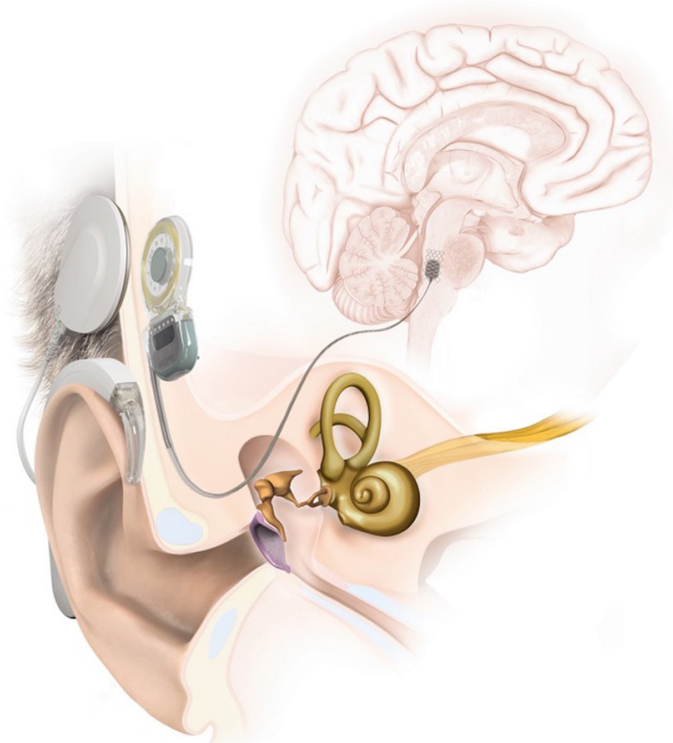

Figure 2 Overview of the position of the implant and placement of the electrode on the cochlear nucleus on the brainstem. Reproduced with permission of MED-EL.

in tertiary tinnitus care. Furthermore, advertisements were placed in magazines and on websites of patients associations and on the research website of the University Medical Center Groningen. Awareness of this study was created by presenting this study protocol at various scientific meetings.

\section{Patient and public involvement}

A plan for organisation of the recruitment of eligible patients was made in consultation and collaboration with a national patients association. Patients were not involved in the development of the research question or in the design of the study. Patient materials, such as information about the study, was screened by the Institutional Review Board (IRB) for understandable not-medical language and approved. Results of this study will be disseminated to study participants and patients via a personal newsletter and via the patients association website.

\section{Study description}

\section{Preoperative phase}

After extensive information on the nature, possible risks and benefits of this study, informed consent is obtained by the study coordinator from eligible patients (for informed consent form, see online supplementary file). When informed consent is obtained, a diagnostic workup is performed. This includes otologic examination, cranial MRI, psychiatric assessment, audiometric and vestibular tests, tinnitus analysis, preoperative assessment by an anaesthesiologist, tinnitus-related and quality of life-related questionnaires and a pregnancy test (if applicable). Whenever an exclusion criterion arises during this diagnostic workup, the patient will be excluded. Otherwise, surgery is scheduled. 


\section{Implantation}

Participants are admitted to the neurosurgical ward of the University Medical Center Groningen for ABI implantation by a trained neurosurgeon. The neurosurgeons are experienced in cerebellopontine angle surgery and were specifically trained for ABI placement. The implant is subperiostally fixated on the parietal skull. Access to the cochlear nucleus is made via retrosigmoid craniotomy. The electrode array is inserted in the lateral recess of the fourth ventricle in the direct vicinity of the cochlear nucleus. The most optimal position of the electrode is determined using a probing electrode with four contact points, applying bipolar stimulation in transverse, longitudinal and oblique directions while recording evoked auditory brainstem responses. After determining the best stimulation site, the active and definitive electrode is placed. With the definite electrode in position, all electrodes are checked for optimal responses. The estimated duration of hospitalisation is 4-6 days.

\section{Postoperative phase}

Shortly postoperative, a CT scan is made to determine the position of the electrode and to screen for intracranial complications. The ABI will be switched on at 6 weeks postoperatively. This happens under monitoring of vital functions, as cranial nerves, such as the vagal nerve, may be stimulated unintentionally. The switch on is performed by a trained medical physicist, using MED-EL software (Maestro V.7.0) and hardware (MAX interface). At this stage, patients receive the external audio processor. At first, the fitting and settings of the $\mathrm{ABI}$ will be aimed at optimising hearing performance, since this approach had given favourable results on tinnitus in earlier implant surgeries for deafness. ${ }^{12}$ Later in the process, other stimulation strategies might be attempted. In the fitting procedure, pitch scaling and consecutive pitch ranking is performed. Electrodes are switched off if they give unwanted side effects during stimulation, for example, facial twitching or dizziness. If electrode stimulation is without complications, further adjustments and fittings can safely take place at the outpatient clinic. Several repetitive fitting sessions will be necessary to find an individual optimal stimulation strategy. In order to get the patient acquainted with the $\mathrm{ABI}$ and to improve their hearing ability, each fitting session is combined with training by a specialised speech therapist.

\section{Outcome measures}

\section{Primary outcome measures}

The primary outcome measure of this study is the change in the score of the Tinnitus Functional Index (TFI) questionnaire. We compare the preoperative (baseline) TFI score with postoperative TFI scores at several time points (figure 3 ), with the primary end point set at 1 year after initial stimulation with the ABI. The TFI consists of 25 items and scores ranging from 0 (no tinnitus complaints) to 100 . The validated Dutch TFI version is used to detect changes in tinnitus outcome after the intervention and its psychometric properties are in line with the original version. ${ }^{16}$ For the Dutch version, no minimal clinical important difference (MCID) was calculated. The MCID in the US version is determined at a 13-point reduction, ${ }^{17}$ however the smallest detectable change in TFI is still debated. $^{18}$

\section{Secondary outcome measures}

Secondary outcome measurements include:

- Audiometric function

- When: preoperatively (baseline) and several time points postoperatively. Audiometric function is determined with the ABI switch on and switched off.

- Measure: determining PTA thresholds and speech audiometry, performed according to guidelines from the Nederlandse Vereniging van Audiologie (Dutch Association of Audiology, www.audiologieboek.nl).

- Important change scores: a change of $>5 \mathrm{~dB}$ is considered as clinically relevant $( \pm 5 \mathrm{~dB}$ is considered measurement error).

Vestibular function

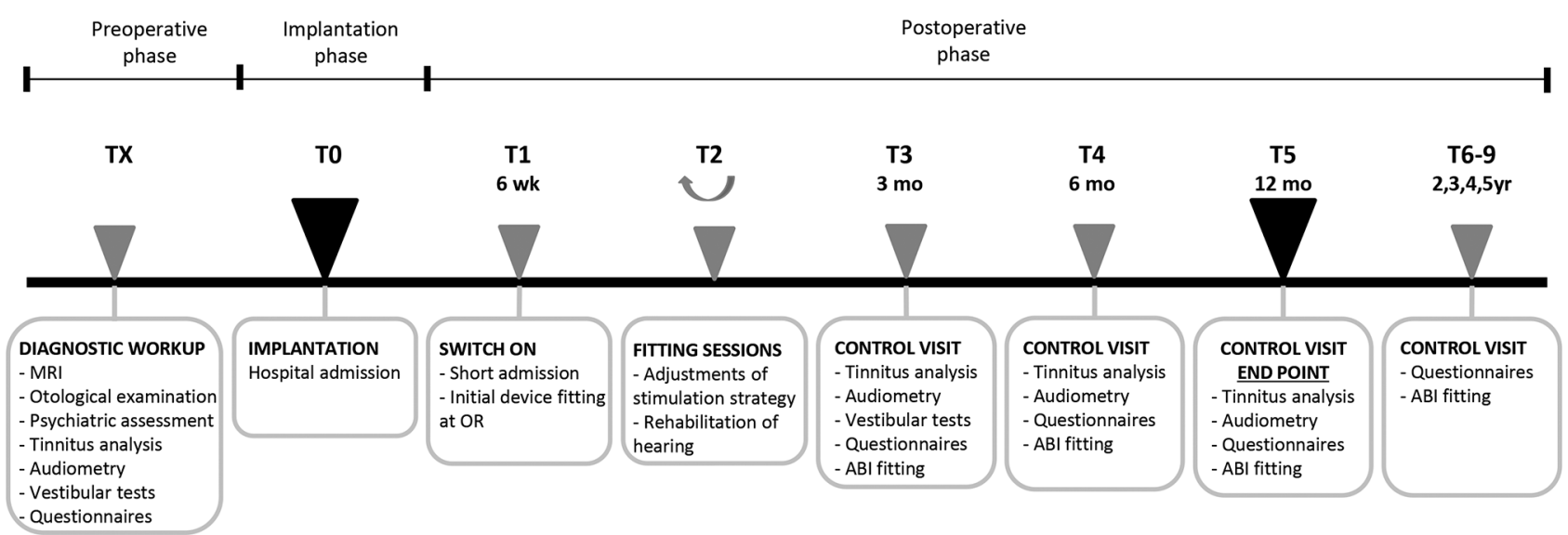

Figure 3 Study timeline. ABI, auditory brainstem implant; OR, operating room; mo, months; T, time point, wk, weeks; yr, years. 
- When: preoperatively (baseline) and at 3 months postoperatively.

- Measure: videonystagmography, rotation tests and calorisation tests of both labyrinths performed according to local hospital protocol.

- Important change scores: clinical relevant changes in vestibular function, that is, newly arisen asymmetrical function during calorisation.

- Tinnitus burden

- When: preoperatively (baseline) and several time points postoperatively.

- Measures:

- Hospital Anxiety and Depression Scale $(\text { HADS })^{19}$ : scores for anxiety/depression range from 0 to a maximum of 21 , with a score $>8$ indicating a possible anxiety/depression.

- Tinnitus Handicap Inventory (THI): scores range from 0 (no tinnitus complaints) to 100 (catastrophic complaints).

- Visual Analogue Scale (VAS) for tinnitus loudness and tinnitus annoyance: patients are instructed to draw a vertical line on a $10 \mathrm{~cm}$ horizontal scale as to how they would rate their tinnitus loudness and annoyance. With 0 being not loud/not annoyed by tinnitus and 100 most thinkable loud/annoyed by tinnitus.

- Important change scores:

- HADS: not calculated for the Dutch version.

- THI: $6-7$ points, ${ }^{20}$ although not calculated for the Dutch version.

- VAS: between 10 and 15 points. ${ }^{21}$

- Tinnitus analysis

- When: preoperatively (baseline) and several time points postoperatively.

- Measure: by tone matching at the contralateral ear (in intensity and frequency), according to guidelines from the Nederlandse Vereniging van Audiologie (Dutch Association of Audiology, www. audiologieboek.nl) .

ABI-related outcomes

- When: several time points after ABI is implanted and switched on.

- Measure:

- Number of electrodes evoking auditory sensation (out of a total of 12 electrodes).

- Pitch matching: frequency matching per electrode using a tone stimulus on the contralateral ear, based on the method for pitch mapping in single-sided deafness with unilateral CIs. ${ }^{22}$

- Tonotopic organisation: tonotopical electrode ordering according to subjective tonal perception, this is performed using the Bubblesort procedure.

- Hours of usage, based on data logging and patient interview.

- Preferred programme (in percentage, out of our programmes).
- When: during the complete course of the study.

- Measure: safety in terms of (serious) adverse events (SAEs), (serious) adverse device effect (SADE).

\section{Follow-up}

Follow-up will take place at 3 and 6 months after switching on the ABI. The end point of this study is set at 12 months. Follow-up will, however, continue yearly, up to 5 years after initial stimulation. An overview of the assessments and their timeline is provided in figure 3.

\section{Data analysis and statistical analysis}

\section{Data management}

All collected data are entered into predesigned electronic case report forms in an Open Clinica database ( www.openclinica.com) by a trained investigator. Data in this database are anonimysed and contains range checks. Stored data in this database are anonymised and password-protected. The database is only accessible by the study coordinator and assigned investigators. All changes made in the database are logged. Hard-copy data will be stored in a locked cabinet. The handling of personal data will comply with the Dutch Personal Data Protection Act. The final dataset will be available to the authors only.

\section{Statistical analysis}

The analysis of data is mainly descriptive. Mean and SD are calculated in case of normally distributed data and median and IQR in case of skewed-distributed data. Differences in the primary outcome measure (ie, TFI) as well as the secondary outcome measures (ie, VAS, THI, HADS) are checked for significance using a paired t-test (if data are normally distributed), although the outcome will be interpreted with caution, since no power calculation was instituted.

SPSS (IBM, version 23) will be used. A p value $<0.05$ is regarded as statistically significant. If needed, analysis will be adjusted for multiple comparisons.

\section{Sample size}

This is a pilot study. Due to the experimental nature of the study, no power analysis was performed. It was empirically decided to select a cohort of 10 patients for this study.

\section{ETHICS AND DISSEMINATION \\ Ethics}

Tinnitus can be very incapacitating, with a large impact on quality of life. Previous reports have shown that the $\mathrm{ABI}$ is a promising method to reduce tinnitus in these patients. Although the major complication rate is low when performed by experienced surgeons, ${ }^{23}$ potential complications can be severe. This study imposes a significant risk on the study participants; it is, however, likely that the potential to ameliorate severely debilitating tinnitus outweighs these risks. This study is performed according to the quality standards of Good Clinical 
Practice. Participation in the study is completely voluntary. Patients can withdraw at any time, without giving any reason. It is stressed that withdrawal does not affect standard clinical care. Written informed consent is obtained from all participants and they are informed when new information arises that may affect their willingness to participate.

The sponsor/investigator has a liability insurance which is in accordance with article 7 of the WMO (Wet Medisch-wetenschappelijk Onderzoek met mensen, that is, Dutch Act for Medical Research Involving Human Subjects). The sponsor has an insurance which is in accordance with the legal requirements in the Netherlands (Article $7 \mathrm{WMO}$ ). This insurance provides cover for damage to research subjects through injury or death caused by the study.

\section{STUDY MONITORING}

This study is monitored by a certified monitor from the Trial Coordination Center, which is independent from the sponsor. Study monitoring includes: checking inclusion and exclusion criteria for included patients, samplewise data checking, correctness of data handling, storage, correctness and completeness of documentation in trial master file, etc. Monitoring will take place after every two to three included patients and after that, once a year for another 4 years.

\section{Safety considerations}

We do not expect a deterioration of hearing due to the implantation. However, because this aspect has not yet been studied, it was decided as a first step to include patients with severe ipsilateral hearing loss (ie, 40 till $90 \mathrm{~dB}$ mean over 1,2 and $4 \mathrm{kHz}$ in PTA). In this patient group, a small loss of hearing sensitivity would not affect daily functioning. Yet, by excluding patients with profound hearing loss $(>90 \mathrm{~dB})$, our study would still be able to quantify unforeseen negative effects on hearing loss. Also, these patients might be eligible for a CI.

Possible complications are mostly related to the ABI surgery. In a study describing such complications, 78 non-tumour patients were analysed..$^{24}$ These patients were not diagnosed with NF2, and therefore are comparable to our patient group. Major complications (meningitis, hydrocephalus, cerebellar contusion) occurred in $6.4 \%$ of cases. No mortality was observed. Minor complications (eg, cerebrospinal fluid leakage, transient hydrocephalus, wound seroma) occurred in $18 \%$. In $30 \%$ of the patients, non-auditory side effects occurred as a result of electrical stimulation. These side effects diminished over time and could be modulated by changing the stimulation settings. ${ }^{24}$ It was concluded that ABI implantation is a safe procedure with a low major complication rate when performed by experienced surgeons. ${ }^{24}$ Inclusion in the study and $\mathrm{ABI}$ implantation are performed consecutively, allowing adequate monitoring of any unforeseen critical event related to the surgery or to the stimulation with the
ABI. Stopping rules are predefined and are described later on in this protocol.

Patients are intensely monitored during the first year following implantation. Patients receive a remote control to switch between four preset stimulation programmes. All of these actions are logged, as well as hours of usage of the implant. Non-auditory side effects and disappointing results on hearing and/or tinnitus will be managed by altering stimulation strategy or, if necessary, by turning off the device. All adverse events (AEs) will be assessed and recorded at each clinical visit. AEs are followed up until they have abated, or until a stable situation has been reached. In case of a SAE or unanticipated SADE (USADE), this will be reported to the IRB 15 days (SAE) or 7 days (USADE) after the first knowledge of the event. Also, a report will be made to the Dutch Health and Youth care Inspectorate.

\section{Stopping rules}

A Data Safety Monitoring Board is not required, due to the small-scale nature of this pilot study and consecutive patient inclusion. Instead, the following 'stopping rules' were predefined:

- If more than onemajor complication occurs in the implanted study population (ie, meningitis, transient hydrocephalus, symptomatic cerebellar contusion).

- If in more than two cases unacceptable worsening of tinnitus is experienced and it is decided to permanently switch off the ABI.

In case one of the stopping rules occurs, the study will be suspended and the risk/benefit balance would be reassessed in accordance with the IRB and/or Dutch Health and Youth care Inspectorate, before considering pursuing the study.

\section{Dissemination and data sharing statement}

The final manuscript will be written by the authors as named above. The results of this study will be published in peer-reviewed journals. Also, findings will be presented at national and international conferences for widespread dissemination of the results. When the trial is finished, data will be available on request.

Contributors PvD, RHF, JMCvD, AM and MJCvdB conceived and designed the study and participated in logistical planning of the study. MJCvdB and AM are responsible for data acquisition. JMCVD, JDMM and RHF perform surgical implantation of the auditory brainstem implants and AM takes care of the perioperative and postoperative fitting sessions. All authors made significant contributions to the development and conceptualisation of the protocol. MJCvdB wrote the manuscript with input from all coauthors. All coauthors reviewed the draft versions of this paper and have read and approved the final manuscript.

Funding This study is investigator-initiated. Funding is provided by MED-EL elektromedizinische Geräte GmbH. All study materials used in this study, that is, implants, software, hardware, monitoring devices, are supplied by MED-EL.

Disclaimer MED-EL has had an advisory role in designing the study. The funding party has no role in the study design and conduct, the collection, management, analysis and interpretation of the data or the preparation and final approval of the manuscript(s). The final manuscript(s) will be send to MED-EL prior to publication for notification.

Competing interests None declared. 
Patient consent for publication Obtained.

Ethics approval This study is approved by the IRB of the University Medical Center Groningen and by the Dutch Health Care Inspectorate.

Provenance and peer review Not commissioned; externally peer reviewed.

Open access This is an open access article distributed in accordance with the Creative Commons Attribution Non Commercial (CC BY-NC 4.0) license, which permits others to distribute, remix, adapt, build upon this work non-commercially, and license their derivative works on different terms, provided the original work is properly cited, appropriate credit is given, any changes made indicated, and the use is non-commercial. See: http://creativecommons.org/licenses/by-nc/4.0/.

\section{REFERENCES}

1. Langguth $B$, Elgoyhen $A B$. Current pharmacological treatments for tinnitus. Expert Opin Pharmacother 2012;13:2495-509.

2. Heller AJ. Classification and epidemiology of tinnitus. Otolaryngol Clin North Am 2003;36:239-48.

3. Savage J, Waddell A. Tinnitus.. Clin Evid 2014;2014:0506.

4. Hobson J, Chisholm E, El Refaie A. Sound therapy (masking) in the management of tinnitus in adults. Cochrane Database Syst Rev 2012;11:CD006371.

5. Martinez-Devesa P, Perera R, Theodoulou M, et al. Cognitive behavioural therapy for tinnitus. Cochrane Database Syst Rev 2010;99:CD005233.

6. Punte AK, Vermeire K, Hofkens A, et al. Cochlear implantation as a durable tinnitus treatment in single-sided deafness. Cochlear Implants Int 2011;12(Suppl 1):S26-S29.

7. Hitselberger WE, House WF, Edgerton BJ, et al. Cochlear nucleus implants. Otolaryngol Head Neck Surg 1984;92:52-4.

8. Edgerton BJ, House WF, Hitselberger W. Hearing by cochlear nucleus stimulation in humans. Ann Otol Rhinol Laryngol Suppl 1982;91(2 Pt 3):117-24.

9. Kaltenbach JA. Summary of evidence pointing to a role of the dorsal cochlear nucleus in the etiology of tinnitus. Acta Otolaryngol Suppl 2006;556:20-6.

10. Brozoski TJ, Bauer CA, Caspary DM. Elevated fusiform cell activity in the dorsal cochlear nucleus of chinchillas with psychophysical evidence of tinnitus. J Neurosci 2002;22:2383-90.

11. Luo H, Zhang X, Nation J, et al. Tinnitus suppression by electrical stimulation of the rat dorsal cochlear nucleus. Neurosci Lett 2012;522:16-20.
12. Soussi T, Otto SR. Effects of electrical brainstem stimulation on tinnitus. Acta Otolaryngol 1994;114:135-40.

13. McSorley A, Freeman SR, Ramsden RT, et al. Subjective outcomes of auditory brainstem implantation. Otol Neurotol 2014.

14. Behr R, Müller J, Shehata-Dieler W, et al. The High Rate CIS Auditory Brainstem Implant for Restoration of Hearing in NF-2 Patients. Skull Base 2007;17:91-107.

15. Roberts DS, Otto S, Chen B, et al. Tinnitus Suppression After Auditory Brainstem Implantation in Patients With Neurofibromatosis Type-2. Otol Neurotol 2017;38:118-22.

16. Rabau S, Wouters K, Van de Heyning P. Validation and translation of the Dutch tinnitus functional index. B-ENT 2014;10:251-8.

17. Meikle MB, Henry JA, Griest SE, et al. The tinnitus functional index: development of a new clinical measure for chronic, intrusive tinnitus. Ear Hear 2012;33:153-76.

18. Folmer RL. Reply to: Psychometric properties of the Tinnitus Functional Index (TFI): Assessment in a UK research volunteer population. Hear Res 2016;335:236.

19. Spinhoven P, Ormel J, Sloekers PP, et al. A validation study of the Hospital Anxiety and Depression Scale (HADS) in different groups of Dutch subjects. Psychol Med 1997;27:363-70.

20. Zeman F, Koller M, Figueiredo R, et al. Tinnitus handicap inventory for evaluating treatment effects: which changes are clinically relevant? Otolaryngol Head Neck Surg 2011;145:282-7.

21. Adamchic I, Langguth B, Hauptmann C, et al. Psychometric evaluation of visual analog scale for the assessment of chronic tinnitus. Am J Audiol 2012;21:215-25.

22. Peters JPM, Bennink E, Grolman W, et al. Electro-acoustic pitch matching experiments in patients with single-sided deafness and a cochlear implant: Is there a need for adjustment of the default frequency allocation tables? Hear Res 2016;342:124-33.

23. Colletti V, Shannon R, Carner M, et al. Outcomes in nontumor adults fitted with the auditory brainstem implant: 10 years' experience. Otol Neurotol 2009;30:614-8.

24. Colletti V, Shannon RV, Carner M, et al. Complications in auditory brainstem implant surgery in adults and children. Otol Neurotol 2010;31:1-564.

25. American Society of Anesthesiologists. ASA physical status classification system. 2014. http://www.google.nl/url?sa=t\&rct= $\mathrm{j} \& \mathrm{q}=$ \&esrc=s\&source=web\&cd=1\&ved=0ahUKEwiGkO7kiuzbAhV QJVAKHYI1CAEQFggqMAA\&url=http\%3A\%2F\%2Fwww.asahq. org \%2F \%2Fmedia\%2Fsites \%2Fasahq\%2Ffiles\%2Fpublic\% 2Fresources $\% 2$ Fstandards-guidelines $\% 2$ Fasa-physical-statusclassification-system.pdf\&usg=AOvVaw2VpwTL1ioJ7-XXfFM7Smwq (Accessed Jul 2016). 


\section{Correction: An auditory brainstem implant for treatment of unilateral tinnitus: protocol for an interventional pilot study}

van den Berge MJC, van Dijk MJMC, Metzemaekers JDM, et al. An auditory brainstem implant for treatment of unilateral tinnitus: protocol for an interventional pilot study. BMJ Open 2019;9:e026185. doi: 10.1136/bmjopen-2018-026185

This article was previously published with an error in Co-author's name. Marc J M C van Dijk was misspelled. The correct name is JMC van Dijk.

Open access This is an open access article distributed in accordance with the Creative Commons Attribution Non Commercial (CC BY-NC 4.0) license, which permits others to distribute, remix, adapt, build upon this work non-commercially, and license their derivative works on different terms, provided the original work is properly cited, appropriate credit is given, any changes made indicated, and the use is non-commercial. See: http://creativecommons.org/licenses/by-nc/4.0/.

(c) Author(s) (or their employer(s)) 2019. Re-use permitted under CC BY-NC. No commercial re-use. See rights and permissions. Published by BMJ.

BMJ Open 2019;9:e026185corr1. doi:10.1136/bmjopen-2018-026185corr1

D) Check for updates 